\title{
La constitucionalización del derecho laboral y su fundamentación
}

\author{
Wilson Yesid Suárez Manrique \\ Corporación Universitaria Rafael Núñez (CURN), Cartagena, Colombia \\ wilsonyesidsuarez@gmail.com
}

\section{RESUMEN}

Este artículo parte de la insatisfacción de la forma cómo ha sido tratado teóricamente el proceso de constitucionalización del derecho laboral. Plantea el análisis de la constitucionalización del derecho laboral desde un proceso de adaptación constituido por cuatro etapas: el impacto, la acomodación, la reestructuración y la devolución. Posteriormente, argumenta que el impulso de la constitucionalización se debe al reconocimiento de los derechos fundamentales inespecíficos, y sugiere una fundamentación directa de los derechos fundamentales laborales desde una perspectiva ontológica, interna e igualitaria, en contraposición con una visión fenomenológica, externa y subjetiva.

Palabras clave: derechos fundamentales; Constitución; derecho laboral; proceso de adaptación y dignidad humana.

Cómo citar: Suárez Manrique, W. Y. (2018). La constitucionalización del derecho laboral y su fundamentación. Ciencias Sociales y Educación, 7(14), 109-125. DOI: https://doi.org/10.22395/csye.v7n14a6

Recibido: 17 de julio de 2018.

Aprobado: 21 de noviembre de 2018. 


\section{The Constitutionalization of Employment Law and its Substantiation}

\section{ABSTRACT}

This article is based upon the dissatisfaction with how the constitutionalization of employment law has been theoretically addressed. It proposes an analysis of the constitutionalization of employment law from an adaptive process based on four stages: the impact, the accommodation (adaptation), the restructuring and its payback. After this, argues that the recent impulse of constitutionalization is due to the recognition of un- specified fundamental rights and suggest a direct substantiation of fundamental employment laws from an ontological perspective, internal and equalitarian, in opposition with a phenomenological, external and subjective vision.

Keywords: fundamental rights; Constitution, employment law; adaptive process; human dignity.

\section{A constitucionalização do direito trabalhista e sua fundamentação}

\section{RESUMO}

Este artigo parte da insatisfação da forma como o processo de constitucionalização do direito trabalhista tem sido tratado teoricamente. Propõe-se a análise da constitucionalização do direito trabalhista a partir de um processo de adaptação constituído por quatro etapas: o impacto, a acomodação, a reestruturação e a devolução. Em seguida, argumenta-se que o impulso da constitucionalização se deve ao reconhecimento dos direitos fundamentais não específicos e sugere-se uma fundamentação direta dos direitos fundamentais trabalhistas sob uma perspectiva ontológica, interna e igualitária, em oposição a uma visão fenomenológica, externa e subjetiva.

Palavras-chave: direitos fundamentais; Constituição; direito trabalhista; processo de adaptação e dignidade humana. 


\section{Introducción}

El impacto de los derechos fundamentales en el derecho laboral se ha convertido en uno de los temas más importantes para el estudio de los constitucionalistas interesados en el derecho laboral. Además, este interés se ha visto acentuado por la práctica judicial, donde, por ejemplo, la Corte Constitucional en las sentencias SU 05 de 2018, SU 057 de 2018, C 636 de 2016 y SU 769 de 2014, ha venido modificando y reconstruyendo algunos de los axiomas legislativos en los cuales se cimentó la construcción de la disciplina laboral a inicios del siglo XX.

Así pues, la Corte Constitucional en la sentencia T-405 de 2007, revisó una acción de tutela instaurada por una trabajadora contra su empleador. La tutelante afirmó que la demandada la citó a su oficina y con base en unas "fotografías inapropiadas", "le dijo si no renunciaba al cargo en la asociación, divulgaría las fotos". Manifestó que, al no acceder a la renuncia, la accionada mostró las fotos a Dagoberto Morales Marriaga, Julio Orozco Payares y al padre de la accionante. La Corte consideró, sobre los hechos del caso "el derecho a la imagen es, sin embargo, un derecho autónomo que puede ser lesionado en forma independiente o concurrente con los derechos a la intimidad, a la honra y al buen nombre de su titular". Entonces, los derechos fundamentales entran a condicionar las relaciones jurídicas que en principio se regían especialmente por la ley y el contrato de trabajo, introduciendo de esta forma nuevos referentes normativos para la solución del problema.

En el caso de esta sentencia se tensionan de forma interesante, en el marco de un contrato de trabajo, situaciones de relevancia constitucional en donde se argumenta con base en derechos fundamentales que tradicionalmente no han estado asociados a los conflictos entre los trabajadores y los empleadores. La anterior referencia es un nicho para la revisión de distintos asuntos laborales y constitucionales. Sin embargo, el principal problema que se desea revisar en este escrito es el debate que denomino los derechos fundamentales en la fábrica y su fundamentación. Este debate corresponde a una especificación de un debate más general que se ha venido dilucidando hace mucho tiempo en la doctrina alemana y que se ha extendido, con matices y marcos diversos, a los debates entre distintos ordenamientos jurídicos: el efecto horizontal de los derechos fundamentales en las relaciones entre particulares (Julio, 2002, p. 125). Este efecto horizontal de los derechos fundamentales es sucedáneo del proceso de constitucionalización del derecho laboral. Proceso que ha sido entendido como una invasión del derecho constitucional en el derecho legislado, que se encarga de tensionar, variar o modificar los elementos ideológicos, sustanciales, procesales y estructurales del derecho positivo.

Pese a la variada doctrina de la constitucionalización del derecho (Bernal, 2006; Calderón, 2011; Carbonell, 2007), por lo general, estas construcciones 
teóricas sufren del problema de un análisis estático. El problema del análisis estático se refiere, en este escrito, a que la constitucionalización se entiende como un fenómeno lineal y poco movible.

En el presente artículo, bajo la concepción de proceso adaptación, se plantea que es factible vislumbrar perspectivas más realistas, móviles, adecuadas y pertinentes de la constitucionalización del derecho laboral. La importancia y la movilidad del proceso de constitucionalización del derecho laboral, se cimienta en la importancia adquirida por los derechos fundamentales inespecíficos y su fundamentación en la dignidad humana.

Para cumplir con lo anterior, el escrito se divide en tres partes. En la primera se presenta el proceso de constitucionalización como un proceso de adaptación constituido por cuatro etapas de análisis: el impacto, la acomodación, la reestructuración, y la devolución. En la segunda, se exhibe la importancia que han adquirido en el ámbito laboral los derechos fundamentales inespecíficos, lo cual condiciona la forma de entender la constitucionalización. En la tercera, se plantea una fundamentación directa de los derechos fundamentales laborales, desde una perspectiva ontológica, interna e igualitaria, en contraposición con una visión fenomenológica, externa y subjetiva.

\section{El proceso de constitucionalización}

El proceso de constitucionalización es un fenómeno rico en matices, perspectivas, tensiones y dinámicas. Uno de los principales problemas que cometen los teóricos (Bernal, 2006; Calderón, 2011; Carbonell, 2007) que abordan el estudio del fenómeno es el de la visión reducida y estática del fenómeno. La constitucionalización es un proceso, cambiante, movible, poco definido, no clasificatorio e inestable. No es una imagen estática que exhibe una situación única. Se trata de una irrupción que muta en diferentes direcciones, que avanza y retrocede. Luego, es difícil de delimitar, de establecer cuáles son sus fronteras y hasta donde llegan sus implicaciones. Resulta arduo decir que un subsistema está o no constitucionalizado, sin entrar a aclarar en qué intensidad y cómo se moviliza.

Una forma de afrontar la visión estática, es decir, lineal y poco movible de la constitucionalización, consiste en entender que se trata de un proceso de adaptación constituido por cuatro etapas: el impacto, la acomodación, la reestructuración, y la devolución. De forma similar a como ha ocurrido en el derecho penal y el derecho de familia, los derechos fundamentales, en el ámbito laboral, en primer lugar, impactan y exigen una reconstrucción de la forma clásica como se entiende determinado subsistema.

El derecho laboral no es inmune a este primer acercamiento con los derechos fundamentales. En el campo laboral resulta muy interesante el impacto efectuado 
por los derechos fundamentales debido a las relaciones de desigualdad que existe entre las partes. La relación entre el trabajador y el empleador, puede caracterizarse, precisamente, por el grado de desigualdad (Sastre, 2001). A diferencia de lo que sucede en la mayoría de las relaciones civiles en las que la igualdad es la regla general, en el derecho laboral la regla general es la asimetría. Este grado de desigualdad exhibe mayores contrastes entre el derecho constitucional y el legislado. Lo anterior se potencia cuando existen poderes laborales muy fuertes, necesidades muy marcadas, y por la progresiva eliminación de las fronteras entre lo público y lo privado.

Este primer momento, modifica, especialmente, a nivel ideológico el subsistema jurídico, al establecer finalidades y mandatos de optimización. Los derechos fundamentales se convierten en una guía ideológica y hermenéutica para entender el derecho positivo. La materialización de este evento se efectúa mediante decisiones jurídicas, leyes y sentencias, en las que se acude a los derechos fundamentales como finalidades mediatas y pautas de interpretación.

Posterior a la entrada en vigencia de los derechos fundamentales en el campo laboral, ocurre un segundo momento de adaptación, en el cual los derechos fundamentales son delimitados o actualizados a través de leyes y sentencias, donde se busca acompasar las exigencias de la vehemencia inicial de los derechos fundamentales y se trata de armonizar el primer impacto. Siguiendo la conocida metáfora de Sebastián Soler (Gargarella, 2009), de la hoja que cae en un lago, al final la rigidez de la hoja, representada por los derechos fundamentales, termina cediendo y convirtiéndose, pese a la diferencia de matices, en una sola con el lago ${ }^{1}$. Bajo este enfoque ilustrativo se representa la adaptación del derecho laboral a los derechos fundamentales.

Luego, en un tercer momento, se genera la etapa de restructuración: se observa cómo los derechos fundamentales no solo se acompasan al orden laboral, sino que, de manera indirecta, modifican sus elementos estructurales. Así como la existencia de un considerable listado de derechos fundamentales y la pretensión de hacerlos normativos, implica el crecimiento de los poderes del juez, los derechos fundamentales en la relación laboral implican un mayor número de deberes por parte del empleador y los compañeros de trabajo, y una mutación de la organización administrativa de la empresa en el tema de libertades laborales y de causales de despido. Es decir, indirectamente los derechos fundamentales ordenan reestructurar las operaciones laborales.

"Soler decía que la llegada de un nuevo artículo a una Constitución o a un Código (él pensaba en el Código Penal), podía verse como la caída de la hoja de un árbol, sobre un lago. Al principio, ambos aparecen como dos cuerpos extraños, ajenos el uno al otro, pero luego las cosas empiezan a cambiar. La hoja cede parte de su firmeza, su textura se hace más suave, y poco a poco pasa a integrarse al lago, y sin desaparecer del todo, queda asociada físicamente con él" Gargarella (2009). 
En un cuarto momento, se exhibe la devolución. Las exigencias de los derechos fundamentales chocan entre ellas mismas, se hacen, en algunos casos, irrealizables, y se tensionan con los derechos fundamentales del empleador. Se implica una especie de equilibrio dinámico en el cual los derechos fundamentales se instauran en la relación laboral. El subsistema del derecho laboral busca equilibrase. Se resalta la importancia de los derechos fundamentales del empleador, y se efectúan restricciones más importantes a los derechos fundamentales del trabajador, como sería el caso de las sentencias de la Corte Constitucional SU 05 de 2018, T-480 de 2016 y T-736 de 2015.

\section{La senda de los derechos fundamentales laborales}

La constitucionalización del derecho laboral se ha repotenciado debido a la importancia que han adquirido los derechos fundamentales inespecíficos. Estos derechos han contribuido a que este fenómeno esté en constante formación y transformación.

\section{Constitucionalismo y derechos inespecíficos}

En el derecho laboral, los derechos fundamentales pueden ser de dos clases generales: específicos o inespecíficos. Los derechos fundamentales laborales específicos, o en sentido estricto, son aquellos derechos fundamentales tipificados en las constituciones de forma directa como derechos laborales. Constituyen especialmente derechos de defensa o de protección que tienen los trabajadores respecto del Estado, el empleador o los particulares. Son ejemplos de estos derechos, la huelga, la libertad sindical, la estabilidad laboral y el derecho a un salario mínimo.

Son garantías generales, derechos humanos, que se establecieron, en un principio, dentro de la legislación laboral (Valverde, 1987) pero, aprovechando el poder político de los trabajadores, sus formas de representación, y teniendo en cuenta las coyunturas constitucionales, se han positivizado en las constituciones como derechos fundamentales laborales (Montoya, 1992). En la Constitución Colombiana están consignados en los artículos 1, 25, 39, 48, 53, 54, 55, 56 y 57.

Estos derechos fundamentales, directamente estatuidos, fueron configurados en cierta clase de Estado. En un principio estos derechos surgieron en el Estado liberal. En el Estado liberal los derechos fundamentales laborales se caracterizaban por consistir en estrategias de libertad como mecanismos para la protección del trabajador de los posibles abusos del Estado (Pérez, 1984). Posterior al constitucionalismo de la Segunda Guerra Mundial estos derechos recibieron importantes aportaciones, ya no solamente desde la perspectiva de los derechos de defensa, sino desde los derechos de prestación. El Estado social de 
derecho tenía como principal finalidad la procura existencial de sus asociados. Luego, en el ámbito laboral se hacen importantes los derechos fundamentales de prestación. A diferencia del anterior estadio en el que se hace importante la libertad, en este modelo teórico se hace importante la igualdad. Y, en especial, la satisfacción de las necesidades básicas del trabajador. Por eso en el primer estadio era importante la libertad sindical, y en el segundo, son relevantes las regulaciones acerca de salarios mínimos y jornadas máximas.

En el marco del constitucionalismo contemporáneo ha tomado importancia inusitada lo que algún sector de la doctrina ha denominado como derechos fundamentales inespecíficos (Sastre, 2001, p. 16). Estos han sido conceptualizados mediante categorías distintas: se ha hablado de ciudadanía del trabajador o derechos fundamentales de la persona del trabajo (Palomeque, 2001, pp. 147-148). En definitiva, puede hablarse en sentido genérico de impregnación constitucional laboral. Se trata de los derechos fundamentales que poseen todas las personas pero que no son específicamente considerados como derechos fundamentales laborales, empero son y pueden ser ejercidos en el marco de una relación laboral. En otras palabras:

Los derechos inespecíficos son otros derechos constitucionales de carácter general y, por ello, no específicamente laborales (que) pueden ser ejercidos, sin embargo, por los sujetos de las relaciones de trabajo (los trabajadores, en particular) en el ámbito de las mismas por lo que en tal caso adquieren un contenido o dimensión laboral sobrevenido. Se produce una 'impregnación laboral' de derechos de titularidad general o inespecífica por el hecho de su ejercicio por trabajadores asalariados (también eventualmente por empresarios) a propósito y en el ámbito de un contrato de trabajo (Cortes, 2004, p. 59)

Así pues, son derechos fundamentales de carácter general, cuya configuración inicial no obedeció a la regulación de las relaciones de los trabajadores con los empleadores. El adjetivo de inespecíficos alude especialmente a que no están configurados con un especial enfoque laboral. Son derechos fundamentales que se diseñaron, especialmente, para la regulación de relaciones entre los ciudadanos y el Estado, lo novedoso consiste en que entran a condicionar de forma variable e inestable las relaciones laborales. Por tanto, "los derechos fundamentales que se reconocen a la persona en su condición como tal y no específicamente en su condición de participante en el proceso productivo pueden ser también ejercitados por los trabajadores en el ámbito de las relaciones de trabajo" (Martín 1997, p. 137).

Si se tratara solo de una diferencia de nombre o de título dentro de los reglamentos constitucionales no sería importante su estudio como una categoría diferente a la de los derechos fundamentales en sentido estricto, pues, estos han sido específicamente estatuidos como derechos laborales, mientras que 
aquellos no. Sin embargo, los derechos fundamentales inespecíficos implican una revolución en la forma como se han entendido los derechos fundamentales en la relación laboral y el proceso de constitucionalización, lo cual conlleva a reconfigurar el contenido, los titulares y la ideología de los derechos en el ámbito laboral. Pues, se estatuyen referentes normativos constitucionales, y personas ajenas a la relación contractual, como los familiares del trabajador o del empleador, tensionan las relaciones laborales.

\section{Principales diferencias}

Existen cinco diferencias de esta clase de derechos con los derechos fundamentales específicamente laborales. La primera, refiere a su nacimiento tardío; la segunda, a la importancia que estos han adquirido en la vida constitucional en las últimas décadas; la tercera, a la titularidad; la cuarta, a la ampliación de los derechos laborales y la quinta, a su protección constitucional ${ }^{2}$.

Pese a que los derechos fundamentales inespecíficos nacen con la positivización constitucional, al igual que los derechos fundamentales laborales en sentido estricto, inclusive antes, su desarrollo jurisprudencial y doctrinal es relativamente tardío en el campo del derecho laboral. Aunque resulte llamativo, los derechos fundamentales que nacieron antes que los derechos fundamentales laborales, vienen a ser significativos en el derecho laboral y en el proceso de constitucionalización, con posterioridad a estos. En este sentido Cortes afirmó que: "si bien el proceso de constitucionalización de los derechos (o libertades) frente al Estado fue anterior al de los derechos laborales, la discusión sobre aquellos en la relación de trabajo ha sido posterior y aún en proceso" (Cortes, 2004, p. 61).

La aparición de estos derechos como catalizadores de la constitucionalización del derecho laboral se debe, especialmente, a dos fenómenos: el aumento de los poderes del empleador en la relación laboral y la ampliación aplicativa de los derechos fundamentales en todos los campos del derecho (Valdés, 2003, p. 69). Los avances tecnológicos incrementan los poderes de vigilancia, control, disciplinarios y económicos, que presuponen un mayor riesgo para los derechos de los trabajadores. Lo cual se complementa con mayores necesidades de los trabajadores en el campo laboral. Es decir, que esta clase de derechos nace ante una mayor amenaza de las libertades básicas de los trabajadores en el marco del nuevo puesto de trabajo. Los casos más emblemáticos de estos derechos se han dado en el ámbito de la intimidad, la honra y la libertad de expresión. Uno de los puntos comunes de estos casos es la utilización por parte del empleador de medios tecnológicos de control.

\footnotetext{
Lo cual, en un grado de abstracción mayor, alude a cuestiones de origen, práctica, titulares, contenido, fuente y exigibilidad. También hay que tener en cuenta las diferencias referidas a la titularidad y el origen. 
El segundo factor alude a la importancia que ha tenido los derechos fundamentales inespecíficos en el proceso de constitucionalización del derecho, en el cual, se tiene como pieza central la aplicación de los derechos fundamentales en gran parte de las relaciones jurídicas, bien sea en el derecho privado (Calderón, 2010), penal (Bernal, 2007) o laboral. Luego, pese a que estos derechos ya estaban consignados en las constituciones, es la conciencia jurídica adquirida mediante el proceso de constitucionalización que trastea las normas fundamentales a las relaciones laborales (Valdés, 2003). Podría decirse, entonces, que estos derechos surgen de una interpretación más profunda, y tal vez extensiva, de los derechos fundamentales. Ello no se fincó exclusivamente en cambios normativos, sino también en una distinta interpretación que permitió redescubrir estos derechos fundamentales. Así, "la discusión de los derechos laborales inespecíficos no proviene de cambios en la normativa constitucional sino en una recreación interpretativa de las mismas normas reconocidas ya hace algún tiempo" (Cortes, 2004).

Las mismas constituciones que reconocen derechos fundamentales laborales reconocen derechos fundamentales generales que tienden a obrar de forma especial en el campo laboral, lo cual viene a afectar la forma de entender la titularidad de estos derechos. Es decir, que los titulares de derechos fundamentales inespecíficos son los mismos titulares de los derechos fundamentales laborales, siempre y cuando sean partícipes de una relación laboral.

Los derechos fundamentales laborales específicos son derechos fundamentales que están diseñados especialmente a favor del trabajador (Valdés, 2003). Entre otras cosas por razones históricas. Los derechos fundamentales en sentido estricto como la huelga, el mínimo vital y móvil, la estabilidad laboral, son derechos que se diseñaron para proteger al trabajador, parte débil del contrato, de las facultades de control y dirección que sobre él ejercía el empleador.

Contrario a esto, los derechos fundamentales inespecíficos son derechos que pueden ser ejercidos no solo por el trabajador sino por el empleador y por personas indirectamente relacionadas al contrato. Piénsese en la hija con síndrome de Down de una trabajadora cuyo contrato laboral es terminado porque debe acompañar a su hija a sus terapias médicas, y debido a la desvinculación la menor queda sin cobertura del sistema de seguridad social en salud. La impregnación de los derechos fundamentales generales al campo de las relaciones laborales no condiciona su uso exclusivo a favor del trabajador.

Así pues, con el entendimiento de estas situaciones se modifica la concepción de los titulares de derechos fundamentales en el contrato de trabajo y el catálogo de derechos. Se amplían de forma importante las fuentes constitucionales de regulación jurídica de los empleadores y trabajadores. A las normas contractua- 
les, legales y constitucionales propiamente laborales hay que sumarle las regulaciones de los derechos fundamentales en un sentido general. Esto hace más abundante y compleja la regulación jurídica al interior del contrato de trabajo.

Los derechos fundamentales, específicos e inespecíficos, de cierta forma amplían los derechos que pueden exigirse al interior del contrato de trabajo en favor de los trabajadores, empero se encuentran limitados, pues los derechos fundamentales no solo se aplican a los trabajadores sino también a los empleadores y demás trabajadores dentro de la empresa. Esto hace compleja su protección y cumplimiento.

La protección de los derechos inespecíficos se sustenta, especialmente, en procedimientos constitucionales. Como se consideran derechos fundamentales, pese a su antigüedad, no fueron concebidos dentro del resorte de las relaciones obrero-patronales, se entiende porque, dentro de la legislación laboral, a diferencia de lo que ocurre con los derechos específicamente laborales, no existen procedimientos especialmente diseñados para su protección. La vía constitucional, mediante acciones de amparo o tutela, ha sido el principal mecanismo de protección de los inespecíficos.

\section{Fundamento de los derechos fundamentales laborales inespecificos}

El proceso de constitucionalización, con sus diversas etapas de reconstrucción, se potencia con la conciencia jurídica de la normatividad de los derechos fundamentales inespecíficos. La forma más plausible de fundamentar estos derechos fundamentales laborales es una perspectiva ontológica, interna e igualitaria de la dignidad humana, en contraposición con una visión fenomenológica, externa y subjetiva.

Los derechos fundamentales laborales son un caso especial de los derechos fundamentales, de forma análoga a como el discurso jurídico es una parte especial del discurso práctico general. Luego, en principio el fundamento de los derechos fundamentales le sirve de soporte. Así las cosas, pueden fundamentarse utilizando las mismas estrategias que han sido utilizadas para fundamentar los derechos fundamentales. La fundamentación de los derechos fundamentales alude a la justificación que soporta su existencia. Desde otra perspectiva: da respuesta a la pregunta de por qué deben existir los derechos fundamentales y otorga un criterio metodológico para dotar de coherencia y sentido las disposiciones normativas que los instituyen.

Si se desea efectuar una fundamentación de los derechos fundamentales como derechos subjetivos se tiene que partir de que éstos protegen posiciones jurídicas individuales. Según Carlos Bernal (2000) "un elemento común a cual- 
quier teoría sobre la fundamentación de los derechos subjetivos consiste en que su punto de partida es siempre una posición del individuo."

La fundamentación de los derechos fundamentales ostenta una cercana relación con el modelo de Estado en el cual se encuentren instituidos. Puede decirse, por ejemplo, que en el Estado liberal, se fundamentan en las facultades morales de Rawls (1996), en el Estado democrático en las concepciones de Habermas (1987), y en el Estado social en los desarrollos de Thugendat (1997) y en los modernos constitucionalismos democráticos, en la concepción de dignidad humana, tal como se explica en párrafos subsecuentes.

Además de la fundamentación independiente, estos derechos pueden ostentar una fundamentación instrumental según esta última, los derechos pueden constituirse como un medio para satisfacer intereses de superior jerarquía. Sin embargo, no se considera adecuada debido a que presupone divisiones entre objetos de los derechos, que desvirtúan le concepción actual de los derechos básicos como un todo.

En el constitucionalismo contemporáneo, desde una posición jurídica individual, la forma más plausible de justificar los derechos fundamentales es la dignidad humana, como atributo inherente a toda persona. Para Francisco Fernández "los derechos fundamentales son inherentes a la dignidad del ser humano y, por lo mismo, se fundan en ella y, a la par, operan como el fundamento último de toda comunidad humana" (2003, p. 3).

La dignidad se encarga de ser la fuente de los derechos fundamentales y el centro del ordenamiento jurídico, alrededor de ella se articulan las relaciones complejas de los derechos fundamentales, de la ley y la jurisprudencia. "Uno de los rasgos sobresalientes del constitucionalismo de la segunda postguerra es la elevación de la dignidad de la persona a la categoría de núcleo axiológico constitucional, y por lo mismo, a valor jurídico supremo del conjunto ordinamental" (Fernández, 2003, p. 3). Los derechos fundamentales son, como estableció Häberle (1994, p. 277), una forma de especificar la dignidad humana, así como la ley y la jurisprudencia son formas de especificar los derechos fundamentales. De Castro ha establecido que "la afirmación de la suprema dignidad de la persona y la convicción de que esa dignidad es raíz y fundamento de los derechos humanos ha llegado a ser ya un tópico de la época actual, con independencia de adscripciones y credos filosóficos o políticos" (1993, p. 123). Por su parte, Pérez Luño, ha afirmado que:

La dignidad humana supone el valor básico fundamentador de los derechos humanos que tienden a explicitar y satisfacer las necesidades de la persona en la esfera moral. De ahí que represente el principio legitimador de los denominados derechos de la personalidad (1984, p. 318). 
Esta fundamentación en la dignidad humana se inspira especialmente en la perspectiva seguida por los instrumentos internacionales y las constituciones contemporáneas. Perspectiva que ha repercutido en los desarrollos teóricos y en las prácticas jurisprudenciales. En el preámbulo y en el artículo $1^{\circ}$ de la Declaración Universal de los Derechos Humanos, en 1948, se estableció, por primera vez en la historia, en un instrumento jurídico la alocución dignidad humana. En el preámbulo se consideró que "la libertad, la justicia y la paz en el mundo tienen por base el reconocimiento de la dignidad intrínseca y de los derechos iguales e inalienables de todos los miembros de la familia humana". En el artículo 1 se preceptuó que "todos los seres humanos nacen libres e iguales en dignidad y derechos y, dotados como están de razón y conciencia, deben comportarse fraternalmente los unos con los otros"3.

Posterior a esta declaración, en otros instrumentos jurídicos internacionales se consagró la dignidad humana como fundamento de los derechos humanos ${ }^{4}$.

El establecimiento de la noción de dignidad humana en la Declaración Universal de los Derechos Humanos fue una de las estrategias mediante la cual se pretendía, principalmente, prevenir las malaventuras del nacismo y sanear los males infringidos. Es decir, la positivización de estos derechos, la creación de convenios internacionales de protección de derechos humanos y el compromiso de las naciones en cuanto a los límites de su soberanía, fueron formas de prevenir y resarcir las consecuencias de la Segunda Guerra Mundial.

La positivización de la dignidad humana a nivel constitucional se representa, por ejemplo, en el artículo 1 de la Constitución Alemana, la cual preceptúa que "la dignidad del hombre es sagrada y constituye deber de todas las autoridades del Estado su respeto y protección". Sin embargo, y pese a su positivización, ninguno de los textos jurídicos reseñados señala una dimensión, noción o definición de la dignidad humana. La dignidad humana es una noción indeterminada

3 Según Eulalia Pascual (2009, p. 17) pese a las construcciones conceptuales de la baja edad media en torno a la dignidad humana, "no fue hasta después de la II Guerra Mundial, y a partir de la Carta de Naciones Unidas, y más concretamente aún de la posterior Declaración de Derechos Humanos de 10 de diciembre de 1948 que se introdujo por primera vez en la historia del Derecho en un texto declarativo o programático la acepción "dignidad" como atributo humano en el que fundamentar un nuevo orden político".

4 De tales instrumentos, los más representativos son: Pactos de Derechos Civiles y Políticos y el de Derechos Económicos, Sociales y Culturales de 19 de diciembre de 1966. El conjunto de estos documentos forma lo que se conoce como Carta de Derechos Humanos. En los preámbulos de ambos pactos, concretamente en su segundo considerando, se efectúa una remisión a la Carta de las Naciones Unidas y a los principios en ella enunciados por los Estados parte en los mismos, señalando a tal efecto que "la libertad, la justicia y la paz en el mundo tienen por base el reconocimiento a la dignidad inherente a todos los miembros de la familia humana y de sus derechos iguales e inalienables, reconociendo que estos derechos se derivan de la dignidad inherente a la persona humana". Se configuran pues los derechos humanos como expresión y concreción sustancial de la idea de dignidad de la persona. 
debido a muchas situaciones ${ }^{5}$. Ha sido abordada por distintas disciplinas tales como la religión, la filosofía, el derecho y la ética (Pascual, 2009, p. 17), además, dentro de cada una de estas disciplinas se ha entendido de distintas maneras (Peces-Barba, 2002, p. 21; González, 2004, p. 417). Así las cosas, la dignidad se considera un concepto indeterminado que, como tal, puede ser interpretado de forma variable de acuerdo con las circunstancias históricas y sociológicas (Pascual, 2009, p. 17), y cada posible interpretación conlleva consecuencias distintas para el ordenamiento jurídico y la protección de los derechos. Por ejemplo, Ruiz (1996, p. 58) ha distinguido cuatro niveles o dimensiones de la dignidad:

1) La dimensión religiosa, en cuya virtud se concibe al hombre a imagen y semejanza de Dios. 2) La dimensión ontológica, en la que se considera al hombre como ser dotado de inteligencia racional, con conciencia de sí mismo y de su superioridad en el orden de la naturaleza, en el orden del mundo. 3) La dimensión ética en el sentido de la autonomía moral, como esencial función de la conciencia valorativa ante cualquier norma y ante cualquier modelo de conducta (esta coincidiría con el planteamiento kantiano). 4) La dimensión social, como estima o fama dimanante de un comportamiento valioso.

La indeterminación semántica de la dignidad humana no es óbice para que no se intente reconstruir un concepto mínimo sobre la dignidad humana que pueda considerarse como un candidato plausible para obtener la fundamentación de los derechos fundamentales laborales en la dignidad humana.

La positivización de la noción no coincide con su nacimiento. Para lograr acercarse a las concepciones contemporáneas de la dignidad humana resulta adecuado partir de como se ha entendido la dignidad humana en épocas pretéritas. Pueden establecerse, de forma general, que desde una perspectiva histórica existen dos líneas generales en las que se ha entendido el término de dignidad (Peces-Barba, 2002, p. 21). En una primera corriente la dignidad se entiende en una concepción social y de reconocimiento público, en la segunda corriente, el acento principal se efectúa en el reconocimiento del ser (Pascual, 2009). Un enfoque puede denominarse fenomenológico, y el otro, ontológico, dependiendo si el acento de la dignidad se encuentra en situaciones externas o internas a las personas.

En el primer caso, en relación con la concepción social y política de la dignidad humana, en Roma, se refería a la nobleza, a la función o labor que se desempeñaba o a las tareas realizadas en favor de los asuntos públicos. Se

5 Parece ser que "la palabra dignidad tiene su origen en el sánscrito, concretamente en la raíz dec, que querría decir ser conveniente, conforme, adecuado a algo o alguien. Posteriormente, fue adoptada por la lengua latina, que le añadió el sufijo -mus, formando el vocablo decmus, que acabó derivando en dignus, que en castellano se convirtió en digno, de donde, a su vez, surgió la palabra dignidad." Beriain (2004). 
trataba de un reconocimiento que la comunidad otorgaba a ciertas personas en razón a sus méritos o a su trabajo, por lo cual, se les concedía una mayor autoridad en la sociedad, y ello implicaba distinguir entre unas personas y otras. Esta forma de concebir la dignidad humana se mantuvo en la edad media, en relación a los nobles y al poder de los reyes y grandes señores. Se dice que la Iglesia también adoptó este sistema en relación con la máxima dignidad del Papa, a la que le seguían la de los cardenales y obispos. Modernamente este concepto se ha utilizado en relación con ciertos cargos públicos de alto rango. En estas situaciones subyace la idea de dignidad asociada a "algo externo a la esencia de la persona". Luego se trata de una noción circunstancial, externa, y de carácter fáctico (Pascual, 2009). Esta perspectiva seria inconveniente para el reconocimiento de los derechos fundamentales de los trabajadores, pues, no sería igualitaria ni equitativa.

Por su parte, la segunda línea narrativa de la dignidad humana se reconstruye desde la óptica estoica y cristiana (Pele, 2001, p. 23). La dignidad no se funda en situaciones externas a las personas, como su trabajo, sino en elementos internos. El hombre se considera el centro del cosmos, bien sea por su semejanza a Dios (Truyol, 1944, p. 97) o por su rango superior en la naturaleza (Pico della Mirandola). El hombre, en resumen, es digno por su naturaleza, con lo cual se viene a fusionar dos conceptos, el de persona y el de dignidad.

Esta concepción de la dignidad, tendrá en el florecimiento del humanismo una revivida vigorosidad (Pérez, 1997, p. 223). Empero, en este estadio, la dignidad tendrá una fuerte relación con el cristianismo de la mano de Tomas de Aquino. La dignidad, se entiende, procede de Dios. Después de diversos vaivenes, en el siglo XVIII, la concepción de la dignidad humana, según Pérez (1997) volverá a ocupar un lugar importante en la filosofía, especialmente, en autores como Wolff y Thomasius. Empero, será Kant (2002), en su Metafísica de las costumbres, quien dote a la dignidad humana de una importancia sin precedentes, en la media que ha sido un referente ulterior para las investigaciones sobre el tema y ha sido objeto de referencias judiciales ${ }^{6}$. Más adelante en la historia, en el pensamiento marxista, se entendió la dignidad como contrapunto de la alienación laboral.

En resumen, pueden verse dos líneas de la dignidad humana: la una revisa la dignidad como "el valor de cada persona, el respeto mínimo a su condición de ser humano, respeto que impide que su vida o su integridad sea sustituida por otro valor social" (de Miguel, 2004, p. 198), y la otra, la entiende en un sentido relacional, esto es, de una cualidad que varía en función de los acontecimientos.

La primera de estas perspectivas resulta plausible para la fundamentación de los derechos fundamentales laborales, mientras que la segunda resulta inconve-

6 En este sentido puede revisarse la sentencia de la Corte Constitucional T 881 de 2002. 
niente. La primera perspectiva es ontológica, igualitaria y puede determinarse por la existencia humana en cuanto sí, sin importar las labores que desarrollen o el cargo que ostenten.

No obstante, lo cierto es que la idea de la dignidad representa algo valioso. Bien sea valioso como una cualidad interna por lo que somos o una cualidad externa por lo que hacemos. La idea de dignidad es relacional con la de valor de la persona humana.

Ahora bien, uno de los elementos que tiene en común las dos concepciones anteriores es que se considera valiosa a una persona, bien sea en un sentido fenomenológico, cuando tiene en cuenta aspectos externos, u ontológico, cuando tiene en cuenta aspectos internos para considerarla digna. Lo valioso es sucedáneo a todo aquello que debe ser conservado y, por ende, no debe ser destruido. Si se destruye es porque no se considera valiosa o porque destrozarla es superior a mantenerla.

Lo valioso puede considerarse en circunstancias externas e internas. En el primer caso, las personas pueden ser consideradas valiosas en dos sentidos, en uno subjetivo y en otro objetivo. En el sentido subjetivo, la persona es valiosa porque se considera valiosa a sí misma. Sin importar que esa valoración pueda ser circunstancial y variable, como cuando una persona se considera importante por llevar a cabo una acción heroica o ejercer determinado trabajo. En un sentido objetivo, las personas se consideran valiosas, no tanto por lo que ellas mismas piensen, sino por lo que consideren las demás personas del colectivo social. Sin embargo, esta forma de valoración tampoco ofrece criterios claros para la estimación. Cada una de estas formas de concebir la valoración puede llevar a conclusiones distintas, y ello conlleva a generar problemas de integración social.

Estas dos formas de valoración conllevan a una misma circunstancia, y es que ambas se fundamentan en los hechos. La valoración de las personas se hace de conformidad con las circunstancias: lo que hace, en lo que trabaja, lo que dice o le sucede. Esta forma de valoración conlleva a estimaciones distintas y a que las consideraciones de la dignidad humana puedan ser ambiguas, luego afectaría de forma inusitada los derechos fundamentales laborales.

Sin embargo, se propone una forma de estimación diferente, que se base en circunstancias internas, que por ende sea más igualitaria y se configure como un candidato plausible para fundamentar los derechos fundamentales laborales. Las personas poseen un valor ontológico por el hecho de ser humanos, se trata de un valor intrínseco que no depende de situaciones externas, ni del trabajo que realicen. Esta forma de otorgar la fundamentación se basa en lo que el hombre es y no en aspectos externos. Este valor es inmutable, igualitario y absoluto de todas las personas y los trabajadores. 
Por esta visión ontológica los trabajadores, dentro del contrato laboral, son titulares tanto de derechos fundamentales específicos e inespecíficos, los cuales estructuran y reestructuran, de forma continua, las obligaciones y los derechos que conforman las relaciones jurídicas laborales.

\section{Agradecimientos}

Artículo de reflexión derivado del proyecto de investigación Derecho constitucional, adelantado dentro de la segunda convocatoria interna de la Corporación Universitaria Rafael Núñez (CURN) de Cartagena (Colombia).

\section{Referencias}

Beriain, I. (2004). Consideraciones sobre el concepto de dignidad humana. Anuario de Filosofía del Derecho, (21), 187-212.

Bernal, C. (2007). El neoconstitucionalismo y la normatividad en el derecho. Bogotá: Universidad Externado de Colombia.

Calderón, J. (2010). La constitucionalización del derecho privado. Bogotá: Uniandes.

Carbonell, M. (2007). Teoría del neoconstitucionalismo, ensayos escogidos. México: Universidad Nacional Autónoma de México.

Corte Constitucional. Colombia. (2018). Sentencia SU-05. M. P. Bernal Pulido, C.

Corte Constitucional. Colombia. (2018). Sentencia SU 057. M.P. Rojas Ríos, A.

Corte Constitucional. Colombia. (2016). Sentencia C 636. M.P. Linares cantillo, A.

Corte Constitucional. Colombia. (2014). Sentencia SU 769. M.P. Palacio Palacio, J.

Corte Constitucional. Colombia. (2007). Sentencia T 405. M.P. Córdova Triviño, J.

Corte Constitucional. Colombia. (2002). Sentencia T 881. M.P. Montealegre Lynett, E.

Cortés, J. (2004). Los derechos inespecíficos laborales: análisis de algunas sentencias del tribunal constitucional. Madrid: Academia de la Magistratura.

De Castro, B. (2004). Los derechos económicos, sociales y culturales. León: Universidad de León.

De Miguel, I. (2004). Consideraciones sobre el concepto de dignidad humana. Madrid: Anuario de Filosofía del Derecho.

Fernández, F. (2003). Estudios constitucionales. México: Universidad Nacional Autónoma de México.

Gargarella, R. (2009). El nuevo constitucionalismo latinoamericano: promesas e interrogantes. Santiago de Chile: Cepal.

González, J. (2004). Autonomía, dignidad y ciudadanía: una teoría de los derechos humanos. Valencia: Tirant lo Blanch

Häberle, P. (2003). La garantía del contenido esencial de los derechos fundamentales (en la Ley Fundamental de Bonn). Madrid: Dykinson. 
Habermas, J. (1987). Teoría de la acción comunicativa: crítica de la razón funcionalista. Madrid: Taurus.

Julio, A. (2000). La eficacia de los derechos fundamentales entre particulares. Bogotá: Universidad Externado de Colombia.

Kant, E. (2002). Fundamentación para una metafísica de las costumbres. Madrid: Alianza Editorial.

Martin, A. (1997). Derecho del trabajo. Madrid: Tecnos.

Montoya, A. (1992). Ideología y lenguaje en las leyes laborales de España (1873-1978). Madrid: Civitas.

Palomeque, M. (2001). Derecho del trabajo. Madrid: Centro de Estudios Ramón Aceres.

Pascual, E. (2009). Configuración jurídica de la dignidad humana en la jurisprudencia del Tribunal Constitucional. Madrid: Bosch.

Peces-Barba, G. (2002). La dignidad de la persona desde la filosofía del derecho. Madrid: Instituto de derechos humanos Bartolomé de las Casas.

Pele, A. (2010). Modelos de la dignidad del ser humano en la Edad Media. Madrid: Universidad Carlos III.

Pérez, A. (1997). Teoría del derecho: una concepción de la experiencia jurídica. Madrid: Tecnos.

Pérez Luño, A. (1984). Derechos humanos, Estado de derechos y Constitución. Madrid: Tecnos.

Rawls, J. (1996). El liberalismo político. Barcelona: Grijalbo-Mondadori.

Ruiz, J. (1996). Derechos fundamentales de la persona. En O. Alzaga (Dir.), Comentarios a las leyes políticas: Constitución española de 1978 (pp. 65-89). Madrid: Edersa

Sastre, R. (2001). Derechos fundamentales de carácter individual y relación de trabajo en la jurisprudencia constitucional española. Bogotá: Pontificia Universidad Javeriana.

Truyol, A. (1944). El derecho y el Estado en San Agustín. Madrid: Editorial Revista de Derecho Privado.

Tugendhat, E. (1997). Lecciones de ética. Barcelona: Gedisa.

Valdés dal-Ré, F. (2003). Los derechos fundamentales de la persona del trabajador: entre la resistencia a su reconocimiento y la reivindicación de su ejercicio. Relaciones Laborales: Revista Crítica de Teoría y Práctica, (2), 69-76.

Valverde, M. (1987). La formación del derecho del trabajo en España. En Congreso de los Diputados (Eds.), La legislación social en la Historia de España: de la revolución liberal a 1936 (pp.157-162). Madrid: Publicaciones del Congreso de los Diputados. 